\title{
E-Bayesian Estimation for Rayleigh Model Using Progressive Type-II Censoring Data
}

\author{
Rashad Mohamed EL-Sagheer \\ Department of Mathematics, Faculty of Science, Al-Azhar University, Nasr City 11884, Cairo, Egypt \\ Rashadmath@yahoo.com
}

Received 11 November 2015

Accepted 23 November 2016

\begin{abstract}
This article deals with using E-Bayesian method under progressively type-II censored sample from the Rayleigh distribution (RD) for computing the estimates of the parameter. The Bayesian and E-Bayesian estimators are obtained under squared error and LINEX loss functions. A comparison between E-Bayesian method and corresponding Bayes and maximum likelihood methods is presented using the Monte Carlo simulation study.

Keywords: Rayleigh distribution; Progressive type-II censoring; Bayesian estimation; E-Bayesian estimation.

2000 Mathematics Subject Classification: 62N05, 62F10
\end{abstract}

\section{Introduction}

The Rayleigh distribution is a special case of the Weibull distribution, which provides a population model useful in several areas of statistics, including life testing and reliability study. A number of authors have considered the problem of estimation of the parameter of Rayleigh distribution using different types of censoring and non-censoring data. Among others, Sinha and Howlader [15] obtained credible and HPD intervals of the parameter and reliability of Rayleigh distribution, Lalitha and Anand [11] studied the modified maximum likelihood estimation for Rayleigh distribution, Fernandez [7] obtained the Bayesian inference from type II doubly censored Rayleigh data, Raqab and Madi [14] considered the estimation of the predictive distribution of the total time on test up to a certain failure in a future sample on the basis of a doubly censored random sample of failure times drawn from a Rayleigh distribution, Soliman and AL-Aboud [17] discussed the Bayesian inference using recored values from Rayleigh Model, Kim and Han [12] discussed estimation of the scale parameter of Rayleigh distribution under general progressive censoring. Lee et al. [13] obtained a Bayes estimator under the Rayleigh distribution with the progressive type II right censored sample. Abou-Elheggag [1] obtained a Bayes estimator under the Rayleigh distribution with the progressive first-failure censored data. 
The probability density function (pdf) and cumulative distribution function (cdf) of the Rayleigh distribution $R D(\theta)$, respectively, given by

$$
f_{X}(x ; \theta)=2 \theta x \exp \left\{-\theta x^{2}\right\} \quad, x>0, \theta>0,
$$

and

$$
F_{X}(x ; \theta)=1-\exp \left\{-\theta x^{2}\right\} \quad, x>0, \theta>0 .
$$

The progressive type-II censoring scheme can be described as follows: Suppose that $n$ units are placed on test and the experimenter decides before hand the quantity $m$, the number of failures to be observed. Now at the time of the first failure, $R_{1}$ of the remaining $n-1$ surviving units are randomly removed from the experiment. At the time of the second failure, $R_{2}$ of the remaining $n-R_{1}-1$ units are randomly removed from the experiment. Finally, at the time of the $m-t h$ failure, all the remaining surviving units $R_{m}=n-m-R_{1}-\cdots-R_{m}-1$ are removed from the experiment. Therefore, a progressive type-II censoring scheme consists of $m$, and $R_{1}, \cdots, R_{m}$ such that $R_{1}+$ $R_{2}+\cdots+R_{m}=n-m$. A recent account on progressive censoring schemes can be obtained in the monograph by Balakrishnan and Aggarwala [3] or in the excellent review article by Balakrishnan [2].

This article can be organized as follows. In Section 2, we present the derivation of the maximum likelihood estimator (MLE) of the involved parameter. Bayesian estimation under squared error and LINEX loss functions is described in Section 3. Section 4 deals with the formulas of E-Bayesian estimation of the parameter under squared error and LINEX loss functions. In Section 5, the properties of E-Bayesian estimation are discussed. Section 6 gives a simulation study. Finally, Section 7 provides some concluding remarks.

\section{Maximum likelihood estimation}

Let $X_{1: m: n}<X_{2: m: n}<\ldots<X_{m: m: n}$ is a progressive type II censored sample from the $R D(\theta)$, with corresponding censoring scheme $R=\left(R_{1}, R_{2}, \ldots, R_{m}\right)$. With progressive type II censoring, $n$ products (or items) are placed on test, the joint density function of all $m$ progressive type II censored order statistic is given by (see Balakrishnan and Aggarwala [3])

$$
f_{x_{1}, x_{2}, \ldots, x_{m}}\left(x_{1}, x_{2}, \ldots, x_{m}\right)=A \prod_{i=1}^{m} f_{X}(x ; \theta)\left[1-F_{X}(x ; \theta)\right]^{R_{i}},
$$

where $x_{i}$ is used instead of $X_{i: m: n}, 0<x_{1}<\ldots<x_{m}<\infty, f_{X}(x ; \theta)$ and $F_{X}(x ; \theta)$ are the pdf and cdf of $X$ as (1.1)-(1.2), respectively. $R \geq 0, i=1,2, \ldots, m$ and

$$
A=n\left(n-1-R_{1}\right)\left(n-2-R 1-R_{2}\right) \ldots\left(n-\sum_{i=1}^{m-1} R_{i}-m+1\right)
$$

Upon substituting (1.1) and (1.2) into (2.1), the likelihood function for $\underline{x}=x_{1}, x_{2}, \ldots, x_{m}$ becomes as

$$
L(\theta ; \underline{x})=A 2^{m} \theta^{m}\left[\prod_{i=1}^{m} x_{i}\right] \exp \left\{-\theta \sum_{i=1}^{m}\left(R_{i}+1\right) x_{i}^{2}\right\},
$$

where $A$ is defined in (2.2), Therefore without the additive constant, the llikelihood function of the observed data can be written as

$$
L(\theta ; \underline{x})=\theta^{m} \exp \{-\theta w\}
$$


where

$$
w=W\left(x_{i}\right)=\sum_{i=1}^{m}\left(R_{i}+1\right) x_{i}^{2} .
$$

The log-llikelihood function can be written as

$$
\ell(\theta ; \underline{x})=m \log (\theta)-\theta w
$$

Thus, the corresponding likelihood equation for the parameter $\theta$ becomes

$$
\frac{\partial \ell(\theta ; \underline{x})}{\partial \theta}=\frac{m}{\theta}-w=0 .
$$

It follows, the ML estimate of $\theta$ is

$$
\widehat{\theta}_{M L}=\frac{m}{w}
$$

where $w$ is as given in (2.5).

\section{Bayesian estimation}

The Bayesian approach provides the possibility for incorporating prior information about the relevant parameters. To this end the parameter $\theta$, is considered as a random variable, having some specified distribution. Here we consider the following gamma conjugate prior density for the parameter $\theta$

$$
\pi(\theta)=\frac{b^{a}}{\Gamma(a)} \theta^{a-1} \exp \{-b \theta\} \quad, \theta>0
$$

where $a>0$ and $b>0$. From (2.4) and (3.1), the posterior density of $\theta$ given $\underline{x}$ can be obtain as

$$
\pi^{*}(\theta \mid \underline{x})=\frac{(b+w)^{(m+a)}}{\Gamma(m+a)} \theta^{m+a-1} \exp \{-\theta(b+w)\} \quad, \theta>0 .
$$

Under the squared error loss function, the Bayes estimate of $\theta$ is given by

$$
\widehat{\theta}_{B S}(a, b)=\frac{m+a}{b+w}
$$

Also, based on LINEX loss function, the Bayes estimate of $\theta$ can be shown to be

$$
\widehat{\theta}_{B L}(a, b)=-\frac{(m+a)}{k} \ln \left(\frac{b+w}{b+k+w}\right) .
$$

For more details about the LINEX loss function, see for example, Calabria and Pulcini [5], Soliman et al. [16] and El-Sagheer [6]. 


\section{E-Bayesian estimation}

According to Han [8], the prior parameters $a$ and $b$ should be selected to guarantee that $\pi(\theta)$ is a decreasing function of $\theta$. The derivative of $\pi(\theta)$ with respect to $\theta$ is

$$
\frac{d \pi(\theta)}{d \theta}=\frac{b^{a}}{\Gamma(a)} \theta^{a-2} \exp \{-b \theta\}[(a-1)-b \theta],
$$

since $a>0, b>0$, and $\theta>0$, it follows $0<a<1, b>0$ due to $\frac{d \pi(\theta)}{d \theta}<0$ and therefore $\pi(\theta)$ is a decreasing function of $\theta$. Assuming that $a$ and $b$ are independent with bivariate density function

$$
\pi(a, b)=\pi_{1}(a) \pi_{2}(b) .
$$

Then, the E-Bayesian estimate of $\theta$ (expectation of the Bayesian estimate of $\theta$ ) can be written as

$$
\widehat{\theta}_{E-B}=E(\theta \mid \underline{x})=\iint \widehat{\theta}_{B}(a, b) \pi(a, b) d a d b,
$$

where $\widehat{\theta}_{B}(a, b)$ is the Bayes estimate of $\theta$ under squared error and LINEX loss functions, given by (3.3) and (3.4) respectively. For more details, see Han [9] or Jaheen and Okasha [10].

\subsection{E-Bayesian estimation using squared error loss function}

E-Bayesian estimation based on three different distributions of the hyperparameters $a$ and $b$ is obtained in this subsection, to investigate the influence of different prior distributions on the EBayesian estimation of $\theta$. The following distributions of $a$ and $b$ may be used

$$
\begin{aligned}
& \pi_{1}(a, b)=\frac{2(c-b)}{c^{2}}, \quad 0<a<1, \quad 0<b<c \\
& \pi_{2}(a, b)=\frac{1}{c}, \quad 0<a<1, \quad 0<b<c \\
& \pi_{3}(a, b)=\frac{2 b}{c^{2}}, \quad 0<a<1, \quad 0<b<c
\end{aligned}
$$

For $\pi_{1}(a, b)$, the E-Bayesian estimate of $\theta$ is obtained from (3.3), (4.3) and (4.4) as

$$
\begin{aligned}
\widehat{\theta}_{E-B S 1} & =\iint \widehat{\theta}_{B S}(a, b) \pi_{1}(a, b) d b d a \\
& =\frac{2}{c^{2}} \int_{0}^{1} \int_{0}^{c}\left(\frac{m+a}{b+w}\right)(c-b) d b d a \\
& =\frac{2\left(m+\frac{1}{2}\right)}{c^{2}}\left[(c+w) \ln \left(1+\frac{c}{w}\right)-c\right] .
\end{aligned}
$$

Similarly, the E-Bayesian estimates of $\theta$ using $\pi_{2}(a, b)$ and $\pi_{3}(a, b)$ are computed and given, respectively, by

$$
\widehat{\theta}_{E-B S 2}=\frac{m+\frac{1}{2}}{c} \ln \left(1+\frac{c}{w}\right),
$$

and

$$
\widehat{\theta}_{E-B S 3}=\frac{2\left(m+\frac{1}{2}\right)}{c^{2}}\left[c-w \ln \left(1+\frac{c}{w}\right)\right] .
$$


where $w$ is as given in (2.5).

\subsection{E-Bayesian estimation using LINEX loss function}

Based on the LINEX loss function, the E-Bayesian estimation of $\theta$ is computed for the three different distributions of the hyperparameters $a$ and $b$ given by (4.4). For $\pi_{1}(a, b)$, the E-Bayesian estimate of $\theta$ is obtained from (3.4), (4.3) and (4.4) as

$$
\begin{aligned}
\widehat{\theta}_{E-B L 1}= & \iint \widehat{\theta}_{B L}(a, b) \pi_{1}(a, b) d a d b \\
= & \frac{2}{c^{2}} \int_{0}^{1} \int_{0}^{c}\left(-\frac{(m+a)}{k}\right) \ln \left(\frac{b+w}{b+k+w}\right)(c-b) d b d a \\
= & \frac{m+\frac{1}{2}}{c^{2} k}\left[c^{2} \ln \left(1+\frac{k}{c+w}\right)-\left(2 c w+w^{2}\right) \ln \left(1+\frac{c}{w}\right)\right. \\
& \left.+\left((k+w)^{2}+2 c(k+w)\right) \ln \left(1+\frac{c}{k+w}\right)-c k\right] .
\end{aligned}
$$

Similarly, the E-Bayesian estimates of $\theta$ based on $\pi_{2}(a, b)$ and $\pi_{3}(a, b)$ are computed and given, respectively, by

$$
\begin{aligned}
\widehat{\theta}_{E-B L 2}= & \frac{m+\frac{1}{2}}{c^{2} k}\left[c^{2} \ln \left(1+\frac{k}{c+w}\right)-c w \ln \left(1+\frac{c}{w}\right)\right. \\
& \left.+c(k+w) \ln \left(1+\frac{c}{k+w}\right)\right],
\end{aligned}
$$

and

$$
\begin{aligned}
\widehat{\theta}_{E-B L 3}= & \frac{m+\frac{1}{2}}{c^{2} k}\left[c^{2} \ln \left(1+\frac{k}{c+w}\right)+w^{2} \ln \left(1+\frac{c}{w}\right)\right. \\
& \left.-(k+w)^{2} \ln \left(1+\frac{c}{k+w}\right)+c k\right] .
\end{aligned}
$$

\section{Properties of E-Bayesian estimation for $\theta$}

This section discuss the relations between $\widehat{\theta}_{E-B S l}(l=1,2,3)$ in Equations (4.5), (4.6) and (4.7), also, the relations between $\widehat{\theta}_{E-B L l}(l=1,2,3)$ in Equations (4.8), (4.9) and (4.10), respectively.

Theorem 5.1. . For E-Bayesian estimator of parameter $\theta$, under squared error loss function, $\left(\widehat{\theta}_{E-B S l}, l=1,2,3\right)$ when $0<c<w$, we have

(i) $\widehat{\theta}_{E-B S 3}<\widehat{\theta}_{E-B S 2}<\widehat{\theta}_{E-B S 1}$.

(ii) $\lim _{w \longrightarrow \infty} \widehat{\theta}_{E-B S 1}=\lim _{w \longrightarrow \infty} \widehat{\theta}_{E-B S 2}=\lim _{w \longrightarrow \infty} \widehat{\theta}_{E-B S 3}$. 
Proof. (i) From (4.5), (4.6) and (4.7) we have

$$
\widehat{\theta}_{E-B S 1}-\widehat{\theta}_{E-B S 2}=\widehat{\theta}_{E-B S 2}-\widehat{\theta}_{E-B S 3}=\frac{2\left(m+\frac{1}{2}\right)}{c^{2}}\left[\frac{c+2 w}{2} \ln \left(1+\frac{c}{w}\right)-c\right]
$$

For $-1<x<1$, we have: $\ln (1+x)=\sum_{i=1}^{\infty}(-1)^{i-1} \frac{x^{i}}{i}=x-\frac{x^{2}}{2}+\frac{x^{3}}{3}-\frac{x^{4}}{4}+\cdots$

Let, $x=\frac{c}{w}$, when $0<c<w$ and $0<\frac{c}{w}<1$, we get :

$$
\begin{aligned}
{\left[\frac{c+2 w}{2} \ln \left(1+\frac{c}{w}\right)-c\right] } & =c\left[\frac{c^{2}}{w^{2}}\left(\frac{1}{3}-\frac{1}{4}\right)+\frac{c^{3}}{w^{3}}\left(\frac{1}{4}-\frac{1}{6}\right)+\frac{c^{4}}{w^{4}}\left(\frac{1}{5}-\frac{1}{8}\right)+\cdots\right] \\
& =c\left[\frac{1}{12} \frac{c^{2}}{w^{2}}+\frac{2}{24} \frac{c^{3}}{w^{3}}+\frac{3}{40} \frac{c^{4}}{w^{4}}+\cdots\right] .
\end{aligned}
$$

According to (5.1) and (5.2), we have $\widehat{\theta}_{E-B S 1}-\widehat{\theta}_{E-B S 2}=\widehat{\theta}_{E-B S 2}-\widehat{\theta}_{E-B S 3}>0$. That is $\widehat{\theta}_{E-B S 3}<\widehat{\theta}_{E-B S 2}<\widehat{\theta}_{E-B S 1}$.

(ii) From (5.1) and (5.2) we get

$$
\begin{aligned}
\lim _{w \longrightarrow \infty}\left(\widehat{\boldsymbol{\theta}}_{E-B S 1}-\widehat{\boldsymbol{\theta}}_{E-B S 2}\right) & =\lim _{w \longrightarrow \infty}\left(\widehat{\boldsymbol{\theta}}_{E-B S 2}-\widehat{\boldsymbol{\theta}}_{E-B S 3}\right) \\
& =\lim _{w \longrightarrow \infty}=\frac{2\left(m+\frac{1}{2}\right)}{c}\left[\frac{1}{12} \frac{c^{2}}{w^{2}}+\frac{2}{24} \frac{c^{3}}{w^{3}}+\frac{3}{40} \frac{c^{4}}{w^{4}}+\cdots\right] \\
& =0 .
\end{aligned}
$$

That is, $\lim _{w \longrightarrow \infty} \widehat{\theta}_{E-B S 1}=\lim _{w \longrightarrow \infty} \widehat{\theta}_{E-B S 2}=\lim _{w \longrightarrow \infty} \widehat{\theta}_{E-B S 3}$. Thus, the proof is complete.

Theorem 5.2. For E-Bayesian estimator of parameter $\theta$, under LINEX loss function, $\left(\widehat{\theta}_{E-B L l}, l=\right.$ $1,2,3)$ when $0<c<w$, we have

(i) $\widehat{\theta}_{E-B L 1}<\widehat{\theta}_{E-B L 2}<\widehat{\theta}_{E-B L 3}$.

(ii) $\lim _{w \longrightarrow \infty} \widehat{\theta}_{E-B L 1}=\lim _{w \longrightarrow \infty} \widehat{\theta}_{E-B L 2}=\lim _{w \longrightarrow \infty} \widehat{\theta}_{E-B L 3}$.

Proof. (i) From (4.8), (4.9) and (4.10) we have

$$
\begin{aligned}
\widehat{\theta}_{E-B L 1}-\widehat{\theta}_{E-B L 2}=\widehat{\theta}_{E-B L 2}-\widehat{\theta}_{E-B L 3} & =\frac{m+\frac{1}{2}}{c^{2} k}\left[\left((k+w)^{2}+c(k+w)\right) \ln \left(1+\frac{c}{k+w}\right)\right. \\
& \left.-\left(c w+w^{2}\right) \ln \left(1+\frac{c}{w}\right)-c k\right] .
\end{aligned}
$$


For $-1<x<1$, we have: $\ln (1+x)=\sum_{i=1}^{\infty}(-1)^{i-1} \frac{x^{i}}{i}=x-\frac{x^{2}}{2}+\frac{x^{3}}{3}-\frac{x^{4}}{4}+\cdots$ Let, $x=\frac{c}{w}$, when $0<c<w$ and $0<\frac{c}{w}<1$, we get :

$$
\begin{aligned}
& \frac{1}{c^{2}}\left[\left((k+w)^{2}+c(k+w)\right) \ln \left(1+\frac{c}{k+w}\right)-\left(c w+w^{2}\right) \ln \left(1+\frac{c}{w}\right)-c k\right] \\
= & \frac{(k+w)^{2}+c(k+w)}{c^{2}}\left[\frac{c}{k+w}-\frac{1}{2} \frac{c^{2}}{(k+w)^{2}}+\frac{1}{3} \frac{c^{3}}{(k+w)^{3}}-\frac{1}{4} \frac{c^{4}}{(k+w)^{4}}+\cdots\right] \\
& -\frac{c w+w^{2}}{c^{2}}\left[\frac{c}{w}-\frac{1}{2} \frac{c^{2}}{w^{2}}+\frac{1}{3} \frac{c^{3}}{w^{3}}-\frac{1}{4} \frac{c^{4}}{w^{4}}+\frac{1}{5} \frac{c^{5}}{w^{5}}+\cdots\right]-\frac{k}{c} \\
= & {\left[\left(\frac{1}{3}-\frac{1}{2}\right) \frac{c}{k+w}+\left(\frac{1}{3}-\frac{1}{4}\right) \frac{c^{2}}{(k+w)^{2}}+\left(\frac{1}{5}-\frac{1}{4}\right) \frac{c^{3}}{(k+w)^{3}}+\cdots\right] } \\
& -\left[\left(\frac{1}{3}-\frac{1}{2}\right) \frac{c}{w}+\left(\frac{1}{3}-\frac{1}{4}\right) \frac{c^{2}}{w^{2}}+\left(\frac{1}{5}-\frac{1}{4}\right) \frac{c^{3}}{w^{3}}+\cdots\right] .
\end{aligned}
$$

According to (5.4) and (5.5), we have:

$\widehat{\theta}_{E-B L 1}-\widehat{\theta}_{E-B L 2}=\widehat{\theta}_{E-B L 2}-\widehat{\theta}_{E-B L 3}<0$. That is $\widehat{\theta}_{E-B L 1}<\widehat{\theta}_{E-B L 2}<\widehat{\theta}_{E-B L 3}$.

(ii) From (5.4) and (5.5) we get

$$
\begin{aligned}
\lim _{w \longrightarrow \infty}\left(\widehat{\theta}_{E-B L 1}-\widehat{\theta}_{E-B L 2}\right)= & \lim _{w \longrightarrow \infty}\left(\widehat{\theta}_{E-B L 2}-\widehat{\theta}_{E-B L 3}\right) \\
= & \frac{m+\frac{1}{2}}{k} \lim _{w \longrightarrow \infty}\left[\left(\frac{1}{3}-\frac{1}{2}\right) \frac{c}{k+w}+\left(\frac{1}{3}-\frac{1}{4}\right) \frac{c^{2}}{(k+w)^{2}}+\cdots\right] \\
& -\frac{m+\frac{1}{2}}{k} \lim _{w \longrightarrow \infty}\left[\left(\frac{1}{3}-\frac{1}{2}\right) \frac{c}{w}+\left(\frac{1}{3}-\frac{1}{4}\right) \frac{c^{2}}{w^{2}}+\cdots\right] \\
= & 0 .
\end{aligned}
$$

That is, $\lim _{w \longrightarrow \infty} \widehat{\theta}_{E-B L 1}=\lim _{w \longrightarrow \infty} \widehat{\theta}_{E-B L 2}=\lim _{w \longrightarrow \infty} \widehat{\theta}_{E-B L 3}$. Thus, the proof is complete.

\section{Monte Carlo simulation study}

We perform Monte Carlo simulation to compare th performaces of the different estimators for different sampling schemes. Monte Carlo simulations were performed utilizing 10000 progressively type-II censored samples for each simulations. The mean squared error (MSE) is used to compear the estimators, where $M S E=\frac{1}{10000} \sum_{i=1}^{10000}\left(\varphi-\hat{\varphi}_{i}\right)^{2}$ and $\hat{\varphi}$ is the estimator of $\varphi$. The samples were generated by using the algorithm described in Balakrishnan and Sandhu [4] using $\theta=0.7683$ with different chooses of $n, m$. We compute the maximum likelihood estimate $\widehat{\theta}_{M L}$ and Bayes estimates $\widehat{\theta}_{B S}, \widehat{\theta}_{B L}$, respectively using (2.8), (3.3) and (3.4). Also, we compute E-Bayesian estimates $\widehat{\theta}_{E-B S 1}, \widehat{\theta}_{E-B S 2}, \widehat{\theta}_{E-B S 3}, \widehat{\theta}_{E-B L 1}, \widehat{\theta}_{E-B L 2}, \widehat{\theta}_{E-B L 3}$ of parameter $\theta$, respectively using, (4.5)-(4.10). Our computational results for the MSEs are computed where the values of the parameters used are $a=0.7329, b=1.5642$ and $c=3, k=2$. All the computions are performed with a Pentium IV processor using Mathematica 8 . We assume that the number of items put on a life test is equal to $n$. Using a progressive type-II censoring scheme, only $m$ observations are obtained from the test. In our study we have used three different censoring schemes (C.S), namely:

Scheme I: $R_{1}=n-m, R_{i}=0$ for $i \neq 1$. 
Scheme II: $R_{\frac{m+1}{2}}=n-m, R_{i}=0$ for $i \neq \frac{m+1}{2}$; if $m$ odd, and $R_{\frac{m}{2}}=n-m, R_{i}=0$ for $i \neq \frac{m}{2}$; if $m$ even. Scheme III: $R_{m}^{2}=n-m, R_{i}=0$ for $i \neq m$.

The computational results are displayed in Table 1.

Table 1. MSE for different estimates of the parameters $\theta$.

\begin{tabular}{|c|c|c|c|c|c|c|c|c|c|c|}
\hline$n, m$ & C.S & $\widehat{\theta}_{M L}$ & $\widehat{\theta}_{B S}$ & $\widehat{\boldsymbol{\theta}}_{E-B S 1}$ & $\widehat{\theta}_{E-B S 2}$ & $\widehat{\theta}_{E-B S 3}$ & $\widehat{\theta}_{B L}$ & $\widehat{\theta}_{E-B L 1}$ & $\theta_{E-B L 2}$ & $\theta_{E-B L 3}$ \\
\hline \multirow[t]{3}{*}{20,10} & I & 0.0701 & 0.0694 & 0.0647 & 0.0651 & 0.0663 & 0.0637 & 0.0625 & 0.0618 & 0.0611 \\
\hline & II & 0.0713 & 0.0699 & 0.0656 & 0.0664 & 0.0672 & 0.0645 & 0.0628 & 0.0623 & 0.0614 \\
\hline & III & 0.0722 & 0.0712 & 0.0662 & 0.0671 & 0.0684 & 0.0656 & 0.0633 & 0.0629 & 0.0625 \\
\hline \multirow[t]{3}{*}{20,15} & I & 0.0685 & 0.0660 & 0.0612 & 0.0625 & 0.0634 & 0.0609 & 0.0595 & 0.0583 & 0.0577 \\
\hline & II & 0.0691 & 0.0667 & 0.0623 & 0.0631 & 0.0642 & 0.0617 & 0.0600 & 0.0594 & 0.0579 \\
\hline & III & 0.0705 & 0.0674 & 0.0635 & 0.0644 & 0.0663 & 0.0625 & 0.0609 & 0.0601 & 0.0586 \\
\hline \multirow[t]{3}{*}{30,20} & I & 0.0523 & 0.0509 & 0.0499 & 0.0502 & 0.0506 & 0.0492 & 0.0476 & 0.0468 & 0.0453 \\
\hline & II & 0.0539 & 0.0519 & 0.0506 & 0.0513 & 0.0527 & 0.0497 & 0.0483 & 0.0477 & 0.0460 \\
\hline & III & 0.0555 & 0.0537 & 0.0517 & 0.0522 & 0.0533 & 0.0506 & 0.0491 & 0.0484 & 0.0468 \\
\hline \multirow[t]{3}{*}{30,25} & I & 0.0410 & 0.0398 & 0.0375 & 0.0382 & 0.0395 & 0.0356 & 0.0341 & 0.0337 & 0.0326 \\
\hline & II & 0.0423 & 0.0415 & 0.0388 & 0.0393 & 0.0402 & 0.0364 & 0.0357 & 0.0340 & 0.0331 \\
\hline & III & 0.0440 & 0.0427 & 0.0395 & 0.0407 & 0.0411 & 0.0376 & 0.0369 & 0.0348 & 0.0340 \\
\hline \multirow[t]{3}{*}{40,30} & I & 0.0374 & 0.0293 & 0.0269 & 0.0273 & 0.0284 & 0.0258 & 0.0239 & 0.0231 & 0.0223 \\
\hline & II & 0.0385 & 0.0299 & 0.0275 & 0.0283 & 0.0291 & 0.0266 & 0.0247 & 0.0236 & 0.0217 \\
\hline & III & 0.0398 & 0.0305 & 0.0281 & 0.0290 & 0.0299 & 0.0273 & 0.0255 & 0.0243 & 0.0228 \\
\hline \multirow[t]{3}{*}{40,35} & I & 0.0222 & 0.0188 & 0.0174 & 0.0179 & 0.0185 & 0.0164 & 0.0159 & 0.0152 & 0.0147 \\
\hline & II & 0.0241 & 0.0196 & 0.0180 & 0.0186 & 0.0194 & 0.0171 & 0.0165 & 0.0159 & 0.0155 \\
\hline & III & 0.0273 & 0.0211 & 0.0192 & 0.0198 & 0.0206 & 0.0185 & 0.0174 & 0.0167 & 0.0161 \\
\hline \multirow[t]{3}{*}{50,40} & I & 0.0115 & 0.0090 & 0.0076 & 0.0082 & 0.0088 & 0.0069 & 0.0061 & 0.0056 & 0.0043 \\
\hline & II & 0.0122 & 0.0096 & 0.0083 & 0.0088 & 0.0094 & 0.0075 & 0.0068 & 0.0061 & 0.0052 \\
\hline & III & 0.0137 & 0.0110 & 0.0099 & 0.0103 & 0.0107 & 0.0092 & 0.0077 & 0.0068 & 0.0060 \\
\hline \multirow[t]{3}{*}{50,45} & I & 0.0053 & 0.0044 & 0.0030 & 0.0035 & 0.0039 & 0.0028 & 0.0023 & 0.0021 & 0.0019 \\
\hline & II & 0.0064 & 0.0058 & 0.0039 & 0.0044 & 0.0053 & 0.0035 & 0.0030 & 0.0026 & 0.0023 \\
\hline & II & 0.0073 & 0.0064 & 0.0047 & 0.0053 & 0.0061 & 0.0043 & 0.0036 & 0.0031 & 0.0028 \\
\hline
\end{tabular}

\section{Concluding remarks}

In this article, we considered the maximum likelihood, Bayesian and E-Bayesian estimates for the parameter of Rayleigh distribution $R D(\theta)$, using progressive type-II censored scheme. Based on the results shown in Table1, we observe the following:

(i) Bayesian and E-Bayesian estimators perform much better than the maximum likelihood estimator in terms of MSEs.

(ii) The MSE of the E-Bayesian estimates of $\theta$ less than the MSE of the Bayesian estimates, so E-Bayesian estimators perform better than the Bayesian estimator.

(iii) The MSE of E-Bayesian estimates under LINEX loss function less than the MSE of E-Bayesian estimates under squared error loss function, so E-Bayesian estimators under LINEX loss function perform better than the E-Bayesian estimator squared error loss function. 
(iv) It is immediate to note that MSE of maximum likelihood, Bayesian and E-Bayesian estimates decrease when $n$ and $m$ increases.

(v) From Table1, comparing the Schemes I and Schemes III, it is clear that the MSEs of the MLEs and Bayes estimators for parameter $\theta$ are greater for the censoring Schemes III than the censoring Schemes I. This may not be very surprising, because the expected duration of the experiments is greater for censoring Schemes I than for the censoring Schemes III. Thus the data obtained by the censoring Schemes I would be expected to provide more information about the unknown parameter than the data obtained by censoring scheme Schemes III.

\section{Acknowledgement}

The author would like to express thanks to editors and the anonymous referees for this valuable comments and suggestions, which significantly improved the paper.

\section{References}

[1] N. A. Abou-Elheggag, Estimation for Rayleigh distribution using progressive first-failure censored data, Journal of Statistics Applications and Probability 2(2) (2013) 171-182.

[2] N. Balakrishnan, Progressive censoring methodology: an appraisal. TEST 16(2) (2007) 211-296.

[3] N. Balakrishnan and R. Aggrawala, Progressive censoring, theory, methods and applications (Birkhauser, Boston, 2000).

[4] N. Balakrishnan and R. A. Sandhu, A simple simulation algorithm for generating progressively type-II censored samples, American Statistics 49 (1995) 229-230.

[5] R. Calabria and G. Pulcini, Point estimation under asymmetric loss functions for left-truncated exponential samples, Communication in Statistics- Theory and Methods 25(3) (1996) 585-600.

[6] R. M. El-Sagheer, Inferences using type-II progressively censored data with binomial removals, Arabian Journal of Mathematics 4 (1) (2015) 127-139.

[7] J. A. Fernandez, Bayesian inference from type-II doubly censored Rayleigh data, Statistics and Probability Letters 48 (2000) 393-399.

[8] M. Han, The structure of hierarchical prior distribution and its applications, Chinese Operation Research and Management Science 6(3) (1997) 31-40.

[9] M. Han, E-Bayesian estimation and hierarchical Bayesian estimation of failure rate, Applied Mathematical Modelling 33 (2009) 1915-1922.

[10] Z. F. Jaheen and H. M. Okasha, E-Bayesian estimation for the Burr type XII model based on type-II censoring, Applied Mathematical Modelling 35 (2011) 4730-4737.

[11] S. Lalitha and M. Anand Modified maximum likelihood estimation for Rayleigh distribution, Соттиnication in Statististics Theory \& Methodes 25 (2) (1996) 389-401.

[12] C. Kim and K. Han, Estimation of the scale parameter of the Rayleigh distribution under general progressive censoring, Journal of the Korean Statistical Society 38 (2009) 239-246.

[13] W. C. Lee, J. W. Wu, M. L. Hong, L. S. Lin and R. L. Chan, Assessing the lifetime performance index of Rayleigh products based on the Bayesian estimation under progressive type-II right censored samples, Journal of Computational and Applied Mathematics 235 (2011) 1676-1688.

[14] M. Z. Raqab and M. T. Madi, Bayesian prediction of the total time on test using doubly censored Rayleigh data, Journal of Statistical Computation and Simulation 72 (2002) 781-789.

[15] S. K. Sinha and H. A. Howlader, Credible and HPD intervals of the parameter and reliability of Rayleigh distribution, IEEE Transaction on Reliability 32 (1983) 217-220.

[16] A. A. Soliman, A. H. Abd Ellah, N. A. Abou-Elheggag and R. M. El-Sagheer, Estimation based on progressive first-failure censored sampling with binomial removals, Intelligent Information Management 5 (2013) 117-125.

[17] A. A. Soliman and F. AL-Aboud, Bayesian inference using recored values from Rayleigh Model with application, European Journal of Operational Research 185 (2008) 659-672. 